Research Paper

\title{
Analyzing Complexity and Fractality of Glucose Dynamics in a Pregnant Woman with Type 2 Diabetes under Treatment
}

\author{
Xiaoyan Chen ${ }^{*}$, Dandan Wang ${ }^{2 *}$, Jinxiang Lin1, Teng Zhang2, Shunyou Deng1, Lianyi Huang1, Yu Jin2, \\ Chang Chen², Zhaozhi Zhang ${ }^{3}$, Jun Zheng ${ }^{2}$, Baoqing Sun ${ }^{4}$, Paul Bogdan ${ }^{5}$, Xiaohua Douglas Zhang ${ }^{2}$ \\ 1. Department of Endocrinology, First Affiliated Hospital of Guangzhou Medical University, Guangzhou 510000, China \\ 2. Faculty of Health Sciences, University of Macau, Taipa 999078, Macau \\ 3. Department of Statistical Science, Duke University, Durham, NC 27708, USA \\ 4. Department of Allergy and Clinical Immunology, Guangzhou Institute of Respiratory Diseases, State Key Laboratory of Respiratory Disease, National \\ Clinical Research Center for Respiratory Disease, First Affiliated Hospital of Guangzhou Medical University, Guangzhou 510000, China \\ 5. Department of Electrical Engineering - Systems, University of Southern California, CA 90089, USA
}

"These two authors contributed equally to this work and should be regarded as Joint First Authors.

$\triangle$ Corresponding author: Xiaohua Douglas Zhang, Email: douglaszhang@um.edu.mo

(C) The author(s). This is an open access article distributed under the terms of the Creative Commons Attribution License (https://creativecommons.org/licenses/by/4.0/). See http://ivyspring.com/terms for full terms and conditions.

Received: 2019.02.03; Accepted: 2019.05.27; Published: 2019.09.07

\begin{abstract}
Currently, the rapid development of continuous glucose monitoring (CGM) device brings new insights into the treatment of diabetic patients including those during pregnancy. Complexity and fractality have recently under fast development for extracting information embodied in glucose dynamics measured using CGM. Although scientists have investigated the difference of complexity in glucose dynamics between diabetes and non-diabetes in order to discover better approaches for diabetes care, no one has analyzed the complexity and fractality of glucose dynamics during the process of adopting CGM to successfully treat pregnant women with type 2 diabetes. Thus, we analyzed the complexity and fractality using power spectral density (PSD), multi-scale sample entropy (MSE) and multifractal detrended fluctuation analysis (MF-DFA) in a clinical case. Our results show that (i) there exists multifractal behavior in blood glucose dynamics; (ii) the alpha stable distribution fits to the glucose increment data better than the Gaussian distribution; and (iii) the "global" complexity indicated by multiscale entropy, spectrum exponent and Hurst exponent increase and the "local" complexity indicated by multifractal spectrum decrease after the successful therapy. Our results offer findings that may bring value to health care providers for managing glucose levels of pregnant women with type 2 diabetes as well as provide scientists a reference on applying complexity and fractality in the clinical practice of treating diabetes.
\end{abstract}

Key words: Continuous glucose monitoring; Complexity analysis; Multiscale sample entropy; Fractal analysis; Type 2 diabetes with pregnancy

\section{Introduction}

Diabetes mellitus is a chronic metabolic disease associated with long-term damage to various organ systems [1]. It causes many complications including cardiovascular diseases, nephropathy, stroke and retinopathy $[1,2]$. Moreover, diabetes with pregnancy is more complicated since hyperglycemia can lead to congenital malformations, preterm delivery, preeclampsia, macrosomia, shoulder dystocia, cesarean delivery and maternal mortality [3]. Currently, the rapid development of continuous glucose monitoring (CGM) device brings new insights into the treatment of diabetic patients including those during pregnancy.

Traditionally, in clinical practice, the information extracted from CGM devices is primarily based on calculations of the percentage of time above and 
below given thresholds, range, and average values of blood glucose, but largely ignores the dynamics of glucose fluctuations $[4,5]$. To extract the information encoded in the dynamical structure of glucose fluctuations, recent research developments in this area employ fractal analysis such as detrended analysis and MSE to assess the complexity of CGM data. Scientists have investigated the complexity of glucose dynamics in type 2 diabetes [4], type 1 diabetes [6], and a mixed pool of type 1 and 2 diabetes [7] against non-diabetics and found that non-diabetic people have a higher complexity than patients with diabetes. However, no one has analyzed the complexity $[4,8-10]$ and fractality $[5,11-16]$ of glucose dynamics during a therapeutic treatment. In this paper, we analyzed the complexity and fractality of glucose dynamics in a pregnant woman with type 2 diabetes during her therapeutic treatment.

\section{Materials and methods}

Nonlinear metrics have been a key category of quantity for extracting information embodied in the dynamics of physiological signals [8-10]. Here we adopted the following three non-linear statistical parameters to analyze the complexity and fractality of glucose dynamics.

\section{Power spectral density}

Fourier transform can convert a time series from time domain to frequency domain and obtain the frequency distribution of the time series. The power spectrum function $|A(f)|^{2}$ represents the power of harmonics with frequency $f$. The PSD of time series describes the distribution of signal power in the frequency domain. For the fractal time series, the relationship between power and frequency is:

$$
|\boldsymbol{A}(\boldsymbol{f})|^{2} \propto \mathbf{c} \cdot \boldsymbol{f}^{-\boldsymbol{\beta}}
$$

where $\mathrm{c}$ is a constant. $\mathrm{c}$ represents the amplitude of the harmonics with frequency $f$ and the $\boldsymbol{\beta}$ is the spectral exponent (the negative slope of the line on the plot of $\log$ power vs. log frequency). For fractal Gaussian noises which are stationary time series, $-\mathbf{1}<\beta<\mathbf{1}$ while for fractal Brownian motions which is non-stationary time series, $<\boldsymbol{\beta}<3$.

The power-law relationship of power and frequency has been found in many physiological time series, such as blood cell perfusion time series [17], heart rate variability [18] and blood glucose. The spectral exponent is the most common parameter for fractal analysis of time series [19]. The method we use to calculate the $\boldsymbol{\beta}$ is lowPSD we which gives the best performance for spectrum analysis [17]. The high-frequency region of spectrum $(\mathrm{fs} / 8<\mathrm{f}<\mathrm{fs} / 2)$ is excluded before the linear fitting of log-log plot.

The power-law relationship of power and frequency has been found in many physiological time series and the spectral exponent has been found to change during different physiological or pathological conditions such as disease and aging $[17,20,21]$.

\section{Multiscale entropy}

Sample entropy is a measure of complexity or irregularity, which was first proposed by Richman and Moorman [22]. The algorithm for calculating sample entropy is as follows. Let $\left\{\boldsymbol{X}_{\boldsymbol{i}}\right\}=\left\{\boldsymbol{x}_{\mathbf{1}}, \ldots, \boldsymbol{x}_{\boldsymbol{i}}, \ldots, \boldsymbol{x}_{\boldsymbol{N}}\right\}$ represent a time series of length N. Define the template vector of length $m$ :

$$
\left\{X_{m}(i)\right\}=\left\{x_{i}, x_{i+1}, x_{i+2}, \ldots, x_{i+m-1}\right\}
$$

and the distance function:

$$
\begin{gathered}
d\left[X_{m}(i), X_{m}(j)\right]=\max _{k}\left\{\left|x_{i+k-1}-x_{j+k-1}\right|\right\}, k= \\
1, \ldots, m \text { (3) }
\end{gathered}
$$

Count the number of vector pairs in template vectors of length $m$ and $m+1$ having $\boldsymbol{d}\left[\boldsymbol{X}_{\boldsymbol{m}}(\boldsymbol{i}), \boldsymbol{X}_{\boldsymbol{m}}(\boldsymbol{j})\right] \leq$ $r$ and denote it by $B$ and $A$, respectively. Then the sample entropy is defined as:

$$
\operatorname{SampEn}(m, r, N)=-\log \frac{A}{B}
$$

where $A$ is the number of template vector pairs having $\boldsymbol{d}\left[\boldsymbol{X}_{\boldsymbol{m + 1}}(\boldsymbol{i}), \boldsymbol{X}_{\boldsymbol{m + 1}}(\boldsymbol{j})\right] \leq \boldsymbol{r}$ of length $m+1$, and $B$ is the number of template vector pairs having of length $\mathrm{m}$. Thus, $\operatorname{SampEn}(m, r, N)$ is the negative natural logarithm of the conditional probability that two sequences similar for $m$ points remain similar at the next point with a tolerance $r$.

With the concept of sample entropy, the process for MSE analysis is as follows. First divide the original signal represented by a time series $\left\{\boldsymbol{X}_{\boldsymbol{i}}\right\}=$ $\left\{\boldsymbol{x}_{1}, \ldots, \boldsymbol{x}_{\boldsymbol{i}}, \ldots, \boldsymbol{x}_{N}\right\}$ into nonoverlapping segments of equal length $(k)$ and calculate the mean value of the data points in each of these segments. This process is called coarse graining, and the newly generated time series is called coarse-grained time series. The length $k$ is called a scale factor. The coarse-graining process is repeated for multiple values of $k$. As the scale factor $k$ changes, we construct different coarse-grained time series, and subsequently we calculate corresponding entropy values on the newly coarse-grained time series. The entropy of coarse-grained time series can be plotted against the scale factor $k$. An $\mathrm{R}$ package for calculating MSE has recently been developed [10]. Here that R package was used to calculate MSE in our clinical case.

\section{Multifractal detrended fluctuation analysis}

The complexity of time series can also be expressed as multifractal behaviors. MF-DFA is a method proposed by Kantelhardt et al. to detect the multifractal behaviors in non-stationary time series. 
MF-DFA explores the scaling behaviors of time series for different values of $q$ which is the order of fluctuation and determines the q-order generalized Hurst exponent $H(q)$. Generally, the smaller $H(q)$ describes the scaling behaviors of large fluctuations, while the larger $H(q)$ describes the scaling behaviors of small fluctuations [23]. When $q=2$, MF-DFA corresponds to the ordinary detrended fluctuation analysis [24]. The $H(2)$ is the scaling exponent of detrended fluctuation analysis and the classical Hurst exponent relates to $H(2)$. The multifractal spectrum $D(q)$ is another measurement of multifractal behaviors and can be obtained via the Legendre transform. The multifractal spectrum will be a single-humped shape with a large arc for multifractal time series. The width of multifractal spectrum $\Delta h$ is calculated by:

$$
\Delta \boldsymbol{h}=\boldsymbol{h}_{\text {max }}-\boldsymbol{h}_{\text {min }}
$$

which can reflect the degree of multifractal behaviors.

The MF-DFA has been proved to be more reliable than other methods of multifractal analysis for time series [25] and there have been a few attempts at applying it to higher dimension [26]. Mukli proposed a new method for multifractal analysis based on MF-DFA [27]. The development of MF-DFA has prompted new applications to complexity of physiological time series [28-31]. Here we use the Matlab code created by Ihlen to analyze the glucose data.

\section{Clinical case}

We observed a 34-year-old woman in early pregnancy who had suffered from both type 2 diabetes and hypertension for 3 years. Her physical examination revealed very high glucose levels (fasting pasma glucose (FPG) $14.3 \mathrm{mmol} / \mathrm{L}$ and glycosylated hemoglobin A1c (HBA1c) 9.6\%) with positive urine ketone and urine protein, but normal levels in estrogen, progesterone and human chorionic gonadotropin. The patient signed the informed consent form.

The patient first received the treatment of multiple dose injection for 7 days without efficacy of glucose control (FPG 10-12.9mmol/L, 2-hour postprandial blood glucose (2hPG) $11-14.4 \mathrm{mmol} / \mathrm{L}$, positive urine ketone). Subsequently, she was treated with a therapy of continuous subcutaneous insulin infusion based on CGM for the first time from 11:55, June 20, 2017 to 08:45, June 23, 2017. This treatment period is referred to as Period 1 throughout the study. The glucose reading from the sensors did not start at 0:00 but at around 12:00. The insulin dose was adjusted after lunch every day based on the glucose readings in the past 24 hours. The basal rates and meal bolus are displayed in Table S1. The method was applied again from June 26 to June 28 because the levels of fasting glucose $(7.9 \mathrm{mmol} / \mathrm{L}$ to $8.4 \mathrm{mmol} / \mathrm{L})$ were still high. This treatment period is referred to as Period 2 throughout the study. After the two periods of treatment, the patient was in good condition, namely normal level of glucose (FPG 5-6.3mmol/L, $2 \mathrm{hPG}$ 6.5-8.5mmol/L, negative urine ketone) and normal blood pressure $(120-130 \mathrm{mmHg} / 70-80 \mathrm{mmHg})$. Moreover, the embryo in the uterus developed well. During the integrative therapy, the diet was not altered.

The CGM based therapy in our case was applied with a device called the MiniMed Paradigm722 real time insulin pump system. This system included an insulin pump, a CGM sensor, a transmitter and a carelink personal software. One new sensor can monitor glucose continuously every five minutes for 3 days. Eight self-monitored blood glucose measurements (three pre-meal, three post-meal, one bedtime and one 3:00) were tested every day. The glucose targets were set for pre-meal, $1 \mathrm{~h}$ post meal and $2 \mathrm{~h}$ post meal. The targeted upper limits [32] were $5.3 \mathrm{mmol} / \mathrm{L}, 7.8 \mathrm{mmol} / \mathrm{L}$ and $6.7 \mathrm{mmol} / \mathrm{L}$ respectively while the lower limit was $3.3 \mathrm{mmol} / \mathrm{L}$ for all glucose targets.

The initial total daily dose was $80 \%$ of the previous dose in the treatment of multiple dose injection. The basal dose and meal bolus were each accounting for approximately $50 \%$ of total daily dose. The three meal boluses were divided using a ratio of 1:1:1 for each meal and results were rounded up to $8 \mathrm{U}$. Six basal rate segments were programmed across a $24 \mathrm{hr}$ period. The average basal rate was approximately one unit per hour. 10\%-20\% adjustment of basal rates and meal bolus was made according to the previous $24 \mathrm{hr}$ sensor glucose and blood glucose. The details of the application of the treatment can be referred to Figure S1 in the Supplementary Materials.

In the MSE calculation, the entropy value is depended on the embedding dimension $\mathrm{m}$ and the tolerance $r$. The selection of $m$ and $r$ can be optimized [33]. Costa et al. have discussed the relationship between MSE and the parameters $m$ and $r$. They obtained the most accurate results when $m=2$ and $r=0.15$ [34]. Therefore, in this calculation, the embedding dimension $\mathrm{m}$ was set as 2 and the tolerance $r$ as 0.15 . When analyzing MF-DFA, in order to satisfy a linear relationship: $\log _{2} F_{q} \sim s c a l e$, we need to choose suitable polynomial $\mathrm{m}$ and scale to get the best linear fit results. Therefore, the order of the fitted polynomial $m$ is set to 2 and the scale is set to $2^{4 \sim} 2^{9}$. 


\section{Results}

\section{Distribution of glucose increments}

The empirical probability density function of the magnitude (absolute value) of the positive blood glucose increments deviates from the Gaussian distribution and is better fitted by an a-stable distribution for either Period 1 or Period 2 (Figure $1 \mathrm{~A} \& \mathrm{C}$ and the left panels of Figure 2). This is also true for the magnitude (absolute value) of the negative blood glucose increments (Figure 1B\&D and the right panels of Figure 2). Therefore, modeling blood sugar dynamics via linear state space models is inadequate and there is a need to analyze the data using non-linear methods such as complexity analysis. Consequently, we applied PSD, MSE and MF-DFA to analyze glucose dynamics in the two treatment periods.

\section{Change of complexity in the clinical case}

The results of PSD analysis on glucose dynamics indicate the existence of power-law relationship between the PSD and frequency in both Periods 1 and

A

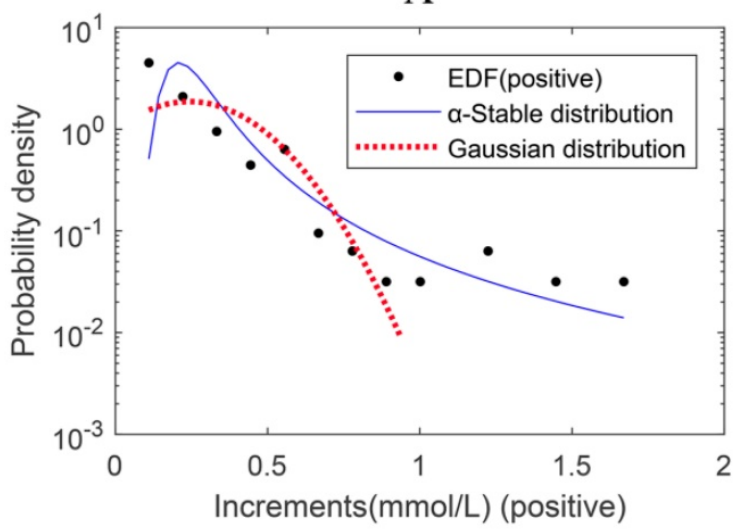

$\mathrm{C}$

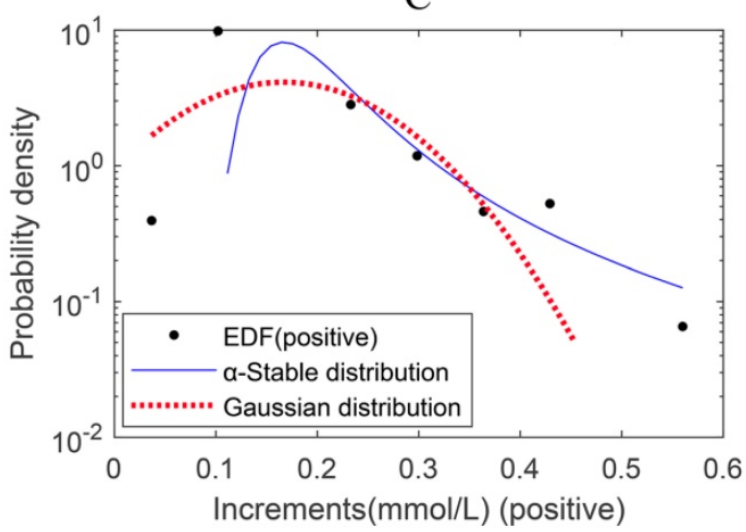

2 (Figure 3A). The spectrum exponent $\beta$ is the negative slope of the linear regression line, $\beta=2$ for Period 1 and $\beta=1.93$ for Period 2, respectively. $\beta>1$ means that the glucose readings are better approximated by a non-stationary fractional Brownian motion [16]. $\beta$ in Period 1 is greater than in Period 2, indicating that the decay rate of the PSD becomes slower after the integrative therapy. According to the relationship between $\beta$ and $H_{f B m}$ (Hurst exponent), $\beta=2 \mathrm{H}_{\mathrm{fBm}}+1$ [35], we can calculate $H_{f B m}$ in both periods. The result shows that $H_{f B m}$ in Period $1(0.87+/-0.019)$ is also larger than that in Period 2 (0.775+/-0.036).

MSE has been applied to explore the complexity of numerous physiological signals [8, 10, 36-38]. Sample entropy is a measure of irregularity and multi-scale sample entropy is a measure of complexity. The sample entropy at each scale in Period 2 is substantially higher than that in Period 1. This indicates that the complexity of glucose dynamics increased in Period 2 compared to Period 1 in the patient (Figure 3B).

B

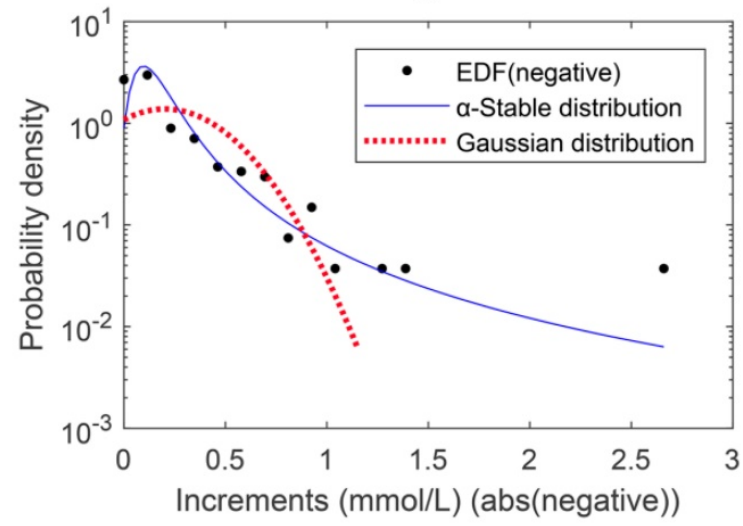

D

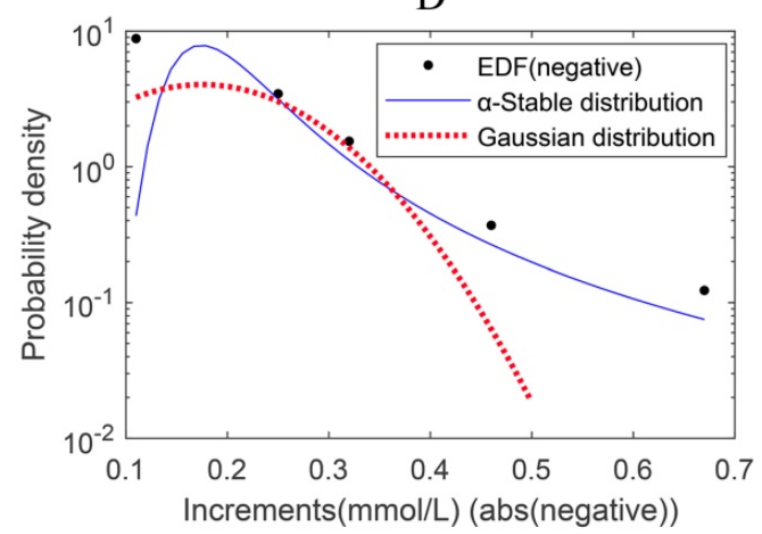

Figure 1. Displaying the results of distribution fitting analysis. (A) Distribution fitting for positive increment of blood glucose values in Period $1, \alpha$-stable (1.1430, 1 , $0.061,0.4945)$ and Gaussian $(0.2428,0.2126)$. (B) Distribution fitting for absolute values of negative increment of blood glucose values in Period $1, \alpha$-stable $(1.0852,1,0.0759$, $0.6886)$ and Gaussian $(0.2020,0.2887)$. (C) Distribution fitting for positive increment of blood glucose values in Period 2, $\alpha$-stable $(1.1565,1,0.0338,0.3126)$ and Gaussian $(0.1668,0.0966)$. (D) Distribution fitting for absolute values of negative increment of blood glucose values in Period $2, \alpha$-stable $(1.1735,1,0.0352,0.3112)$ and $G$ aussian $(0.1752$, 0.0989). EDF: empirical density function. 

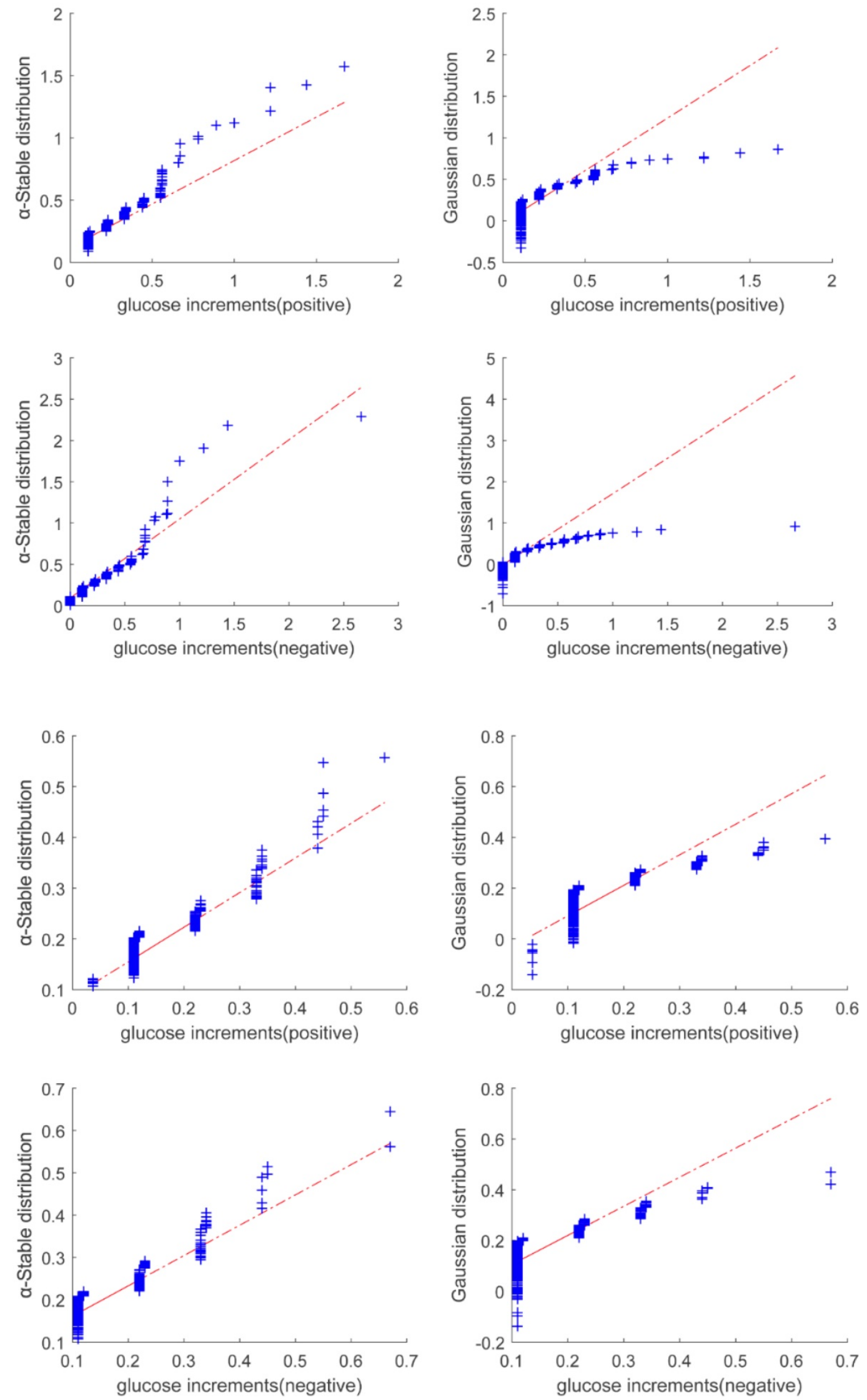

Figure 2. Quantile-quantile plot of glucose increments for Period 1 (Top two panels) and Period 2 (bottom two panels). The figure shows that the alpha stable distribution (shown in the left panels) fits to the data better than Gaussian distribution (shown in the right panels). 

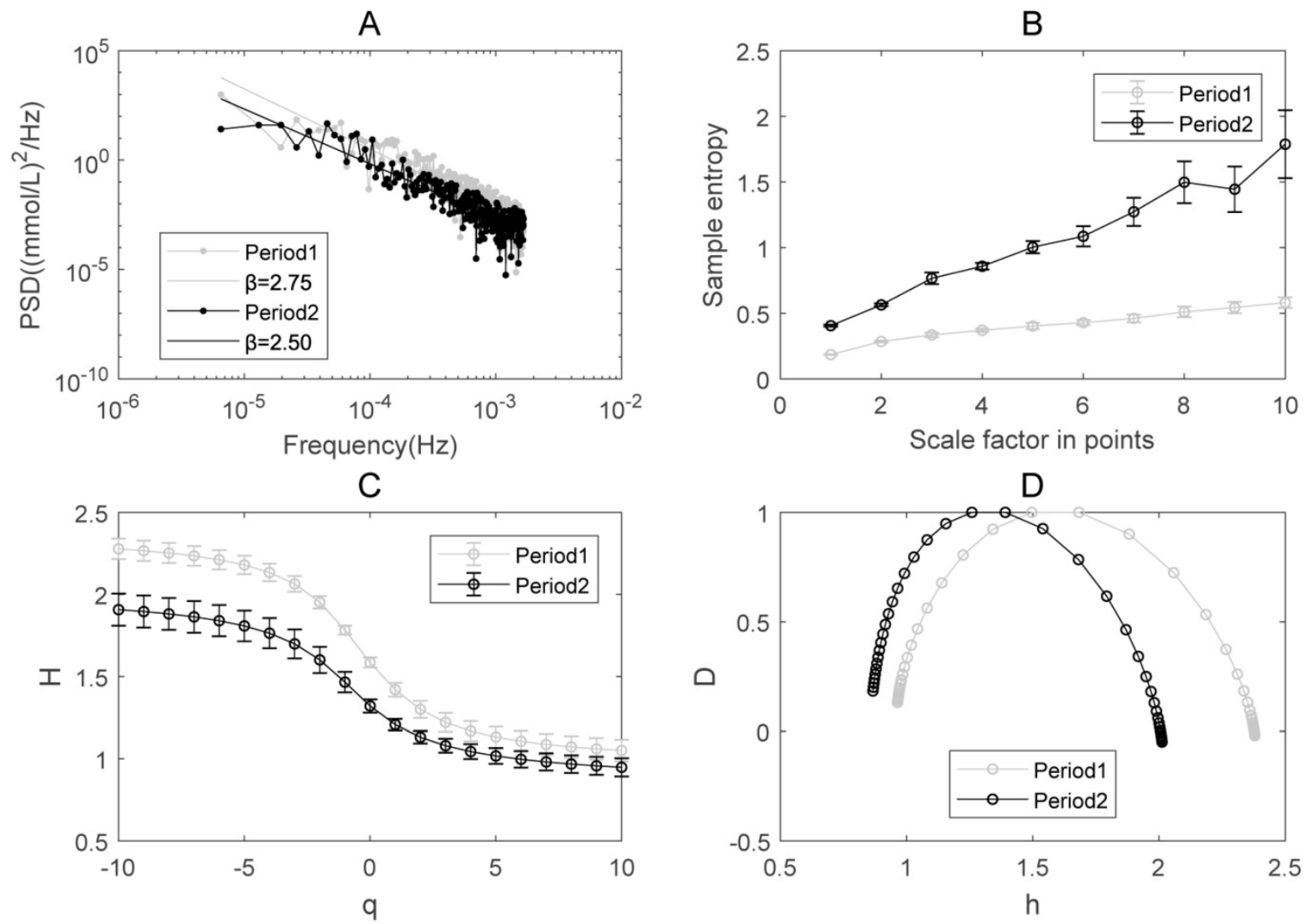

Figure 3. The complexity of glucose dynamics in Periods 1 and 2 for the integrative treatment. (A) The power spectral density. (B) Multiscale entropy analysis (MSE). (C) Multifractal detrended fluctuation analysis: Q-order Hurst exponent. (D) Multifractal spectrum analysis. The error bar (i.e., standard deviation) in Panels B and C was given by bootstrapping all the ordered glucose time series that contain $95 \%$ of the original data.

The results of MF-DFA applied to the glucose dynamics indicates that the generalized Hurst exponent in Period 1 is greater than that in Period 2 (Figure 3C). The higher the Hurst exponent, the lower the fractal dimension, and the lower the global complexity accordingly in certain circumstances [39]. The overall decrease of $\mathrm{H}(\mathrm{q})$, which is consistent with the results of PSD, indicates that the global complexity increased after the integrative therapy.

The single-humped shape of the multifractal spectrum further illustrates the existence of multifractal behavior in blood glucose dynamics (Figure 3D). The width of the multifractal spectrum $\Delta \mathrm{h}$ is a measure of multifractality. Different from the sample entropy and Hurst exponent, $\Delta$ h reflects the "local" fractality and complexity of time series [27]. The width of the multifractal spectrum $\Delta \mathrm{h}$ in Period 1 (i.e., 1.32) is greater than in Period 2 (i.e., 1.12), indicating that the degree of multifractality and the "local" complexity become smaller after treatment.

\section{Discussion}

Based on the data of glucose dynamics measured by CGM, we analyzed complexity and fractality of a pregnant woman with type 2 diabetes that was treated successfully with continuous subcutaneous insulin infusion and CGM, and compared the results in two treatment periods. After this integrative treatment, the glucose level was reduced to a normal range and became stable. The complexity analysis shows that (i) the Spectrum exponent $\beta$ (the negative slope of the linear regression line in Figure 3A) decreased; (ii) the multiscale entropy increased (Figure 3B); (iii) the Hurst exponent decreased (figure 3C) and (iv) the width of the multifractal spectrum decreased (Figure 3D) in Period 2 as compared to Period 1. These results indicated that the "global" complexity indicated by multiscale entropy, spectrum exponent and Hurst exponent increased; and the "local" complexity indicated by multifractal spectrum decreased after a successful treatment on diabetes.

To the best of our knowledge, this is the first time that an increased "global" complexity in glucose dynamics during the treatment periods for diabetes was displayed in a clinical case where a pregnant woman with type 2 diabetes was treated using continuous subcutaneous insulin infusion along with CGM. Taking into consideration other studies having shown that the complexity of blood glucose systems is higher in those without diabetes compared to patients with diabetes [4, 6, 7], this gives us increased confidence that complexity (global or local) has the 
potential of serving as an effective index to assess efficacy in the treatment of diabetes, as well as potential measures for assessing the therapeutic efficacy of various control algorithms for artificial pancreas devices. That is, the Spectrum exponent $\beta$ in PSD analysis, the sample entropy in MSE analysis, Hurst exponent $\mathrm{H}(\mathrm{q})$ and the width of the multifractal spectrum $\Delta \mathrm{h}$ in MF-DFA all have the potential of being used as the indicator of treatment adjustment in diabetes.

Another important observation is that medical cyber-physical systems should not only aim to control for ensuring specific reference values, but also to preserve a higher degree of global complexity of physiological processes which intrinsically encapsulates the capabilities of sustaining large perturbations and adapting to environmental influences [5]. The observed changes of complexity in the pregnant woman with type 2 diabetes may serve as inspiration to medical cyber physical systems design and in particular to artificial pancreas design [5]. More precisely, the control algorithms in these artificial pancreas devices may consider not only the blood glucose reference values, but also measures of complexity. It has been verified that the nonlinear optimal controller based on fractal calculus concepts is superior to nonfractal controllers in the artificial pancreas design [5]. The nonlinear analysis based on complexity may help artificial pancreas devices to control the blood glucose more tightly and prevent medical complications.

In this paper, we used MSE, PSD and MF-DFA to measure the complexity of glucose data. The three methods are measurement of complexity from different aspects. MSE is a method to measure complexity through irregularity across different scales. Higher MSE value means higher complexity. By contrast, PSD and MFDFA are the methods to measure complexity through fractal behavior (A higher Hurst exponent corresponds to a lower fractal dimension, and higher Hurst exponent means lower complexity accordingly [39]). In addition, the scale for PSD analysis is frequency and we exclude the high-frequency region of spectrum $(\mathrm{fs} / 8<\mathrm{f}<\mathrm{fs} / 2)$, where $\mathrm{fs}$ is sampling frequency, while the scale for MFDFA spans the time axis and the scale ranges are $\left(2^{\wedge} 4 \sim 2^{\wedge} 9\right)$. It means that PSD is a method for fractal analysis in frequency domain and MFDFA is a method for multifractal analysis in time domain. There may be a need to explore whether and when one method is better than others for future research.

Still there are undoubtedly certain limitations to our study. To be able to obtain reliable MSE values and fractal analysis, a large number of observed data points (usually at least a thousand) are required.
Furthermore, it has recently been shown [40] that sample entropy is equivalent to conditional entropy quantified by Heaviside kernel function and depends on the properties of correlation and the type of artifacts in the signal. Future research should be conducted to explore the auto-correlation properties of glucose dynamics data and to explore the type of artifacts in the data and its impact on non-linear parameters like sample entropy.

\section{Abbreviations}

CGM: continuous glucose monitoring; MSE: multi-scale sample entropy; PSD: power spectral density; MF-DFA: multifractal detrended fluctuation analysis; FPG: fasting plasma glucose; 2hPG: 2 hours postprandial blood glucose; HbA1c: glycosylated hemoglobin A1c.

\section{Supplementary Material}

Supplementary methods, figures, and table. http://www.ijbs.com/v15p2373s1.pdf

\section{Acknowledgments}

This work was supported by University of Macau through Research Grants MYRG201800071-FHS and FHS-CRDA-029-002-2017. P.B. gratefully acknowledges the support by the National Science Foundation under CAREER Award CPS-1453860, the U.S. Army Defense Advanced Research Projects Agency (DARPA) under grant no. W911NF-17-1-0076, and DARPA Young Faculty Award under grant no. N66001-17-1-4044.

\section{Competing Interests}

The authors have declared that no competing interest exists.

\section{References}

1. Alam U, Asghar O, Azmi S, Malik RA. General aspects of diabetes mellitus. Handbook of clinical neurology. 2014; 126: 211-22.

2. Horr S, Nissen S. Managing hypertension in type 2 diabetes mellitus. Best practice \& research Clinical endocrinology \& metabolism. 2016; 30: 445-54.

3. Castorino K, Paband R, Zisser H, Jovanovic L. Insulin pumps in pregnancy: using technology to achieve normoglycemia in women with diabetes. Curr Diab Rep. 2012; 12: 53-9.

4. Costa MD, Henriques T, Munshi MN, Segal AR, Goldberger AL. Dynamical glucometry: use of multiscale entropy analysis in diabetes. Chaos (Woodbury, NY). 2014; 24: 033139.

5. Ghorbani M, Bogdan P. A cyber-physical system approach to artificial pancreas design. 2013 International Conference on Hardware/Software Codesign and System Synthesis (CODES+ISSS). IEEE. 2013;:1-10.

6. Crenier L, Lytrivi M, Van Dalem A, Keymeulen B, Corvilain B. Glucose Complexity Estimates Insulin Resistance in Either Nondiabetic Individuals or in Type 1 Diabetes. The Journal of clinical endocrinology and metabolism. 2016; 101: 1490-7.

7. Chen JL, Chen PF, Wang HM. Decreased complexity of glucose dynamics in diabetes: evidence from multiscale entropy analysis of continuous glucose monitoring system data. American journal of physiology Regulatory, integrative and comparative physiology. 2014; 307: R179-83.

8. Zhang XD, Pechter D, Yang L, Ping X, Yao Z, Zhang R, et al. Decreased complexity of glucose dynamics preceding the onset of diabetes in mice and rats. PloS one. 2017; 12: e0182810.

9. Zhang XD. Entropy for the Complexity of Physiological Signal Dynamics. Advances in experimental medicine and biology. 2017; 1028: 39-53. 
10. Zhang $\mathrm{XD}$, Zhang $\mathrm{Z}$, Wang D. CGManalyzer: an $\mathrm{R}$ package for analyzing continuous glucose monitoring studies. Bioinformatics. 2018; 34: 2340.

11. Ivanov PC, Amaral LA, Goldberger AL, Havlin S, Rosenblum MG, Struzik ZR, et al. Multifractality in human heartbeat dynamics. Nature. 1999; 399: 461-5.

12. Nunes Amaral LA, Ivanov PC, Aoyagi N, Hidaka I, Tomono S, Goldberger $\mathrm{AL}$, et al. Behavioral-independent features of complex heartbeat dynamics. Physical review letters. 2001; 86: 6026-9.

13. Ivanov PC, Nunes Amaral LA, Goldberger AL, Havlin S, Rosenblum MG, Stanley HE, et al. From $1 / \mathrm{f}$ noise to multifractal cascades in heartbeat dynamics. Chaos (Woodbury, NY). 2001; 11: 641-52.

14. Ivanov PC, Chen $\mathrm{Z}, \mathrm{Hu} \mathrm{K}$, Eugene Stanley $\mathrm{H}$. Multiscale aspects of cardiac control. Physica A: Statistical Mechanics and its Applications. 2004; 344: 685-704.

15. Goldberger AL, Amaral LAN, Hausdorff JM, Ivanov PC, Peng CK, Stanley HE. Fractal dynamics in physiology: Alterations with disease and aging. Proceedings of the National Academy of Sciences of the United States of America. 2002; 99: 2466-72.

16. Eke A, Herman P, Kocsis L, Kozak LR. Fractal characterization of complexity in temporal physiological signals. Physiological measurement. 2002; 23: R1-38.

17. Eke A, Herman P, Bassingthwaighte JB, Raymond GM, Percival DB, Cannon $\mathrm{M}$, et al. Physiological time series: distinguishing fractal noises from motions. Pflugers Archiv : European journal of physiology. 2000; 439: 403-15.

18. Sakata S, Hayano J, Mukai S, Okada A, Fujinami T. Aging and spectral characteristics of the nonharmonic component of 24-h heart rate variability. The American journal of physiology. 1999; 276: R1724-31.

19. Weissman A, Binah O. The fractal nature of blood glucose fluctuations. Journal of Diabetes and its Complications. 2014; 28: 646-51.

20. Castiglioni P, Di Rienzo M, Radaelli A. Effects of autonomic ganglion blockade on fractal and spectral components of blood pressure and heart rate variability in free-moving rats. Autonomic neuroscience : basic \& clinical. 2013; 178: 44-9.

21. Weiss B, Clemens Z, Bodizs R, Halasz P. Comparison of fractal and power spectral EEG features: effects of topography and sleep stages. Brain research bulletin. 2011; 84: 359-75.

22. Richman JS, Moorman JR. Physiological time-series analysis using approximate entropy and sample entropy. American journal of physiology Heart and circulatory physiology. 2000; 278: H2039-49.

23. Ihlen E. Introduction to Multifractal Detrended Fluctuation Analysis in Matlab. Frontiers in physiology. 2012; 3:141

24. Peng CK, Buldyrev SV, Havlin S, Simons M, Stanley HE, Goldberger AL. Mosaic organization of DNA nucleotides. Physical Review E. 1994; 49: 1685-9.

25. Kantelhardt JW. Fractal and Multifractal Time Series. In: Meyers RA, editor. Mathematics of Complexity and Dynamical Systems. New York, NY: Springer New York. 2011;:463-87.

26. Zhou Y, Leung Y, Yu Z-G. Relationships of exponents in two-dimensional multifractal detrended fluctuation analysis. Physical Review E. 2013; 87: 012921.

27. Mukli P, Nagy Z, Eke A. Multifractal formalism by enforcing the universal behavior of scaling functions. Physica A: Statistical Mechanics and its Applications. 2015; 417: 150-67.

28. Thomas F, Signal M, Chase JG. Using Continuous Glucose Monitoring Data and Detrended Fluctuation Analysis to Determine Patient Condition: A Review. Journal of diabetes science and technology. 2015; 9: 1327-35.

29. Zorick T, Mandelkern MA. Multifractal detrended fluctuation analysis of human EEG: preliminary investigation and comparison with the wavelet transform modulus maxima technique. PloS one. 2013; 8: e68360.

30. Gieraltowski J, Zebrowski JJ, Baranowski R. Multiscale multifractal analysis of heart rate variability recordings with a large number of occurrences of arrhythmia. Physical review E, Statistical, nonlinear, and soft matter physics. 2012; 85: 021915.

31. Dutta S, Ghosh D, Chatterjee S. Multifractal detrended fluctuation analysis of human gait diseases. Frontiers in physiology. 2013; 4: 274.

32. Management of Diabetes in Pregnancy: <em>Standards of Medical Care in Diabetes - 2018</em>. Diabetes care. 2018; 41: S137-S43.

33. Lake DE, Richman JS, Griffin MP, Moorman JR. Sample entropy analysis of neonatal heart rate variability. Am J Physiol Regul Integr Comp Physiol. 2002; 283: R789-97.

34. Costa M, Goldberger AL, Peng CK. Multiscale entropy analysis of biological signals. Phys Rev E Stat Nonlin Soft Matter Phys. 2005; 71: 021906

35. Eke A, Hermán P, Bassingthwaighte J, Raymond G, Percival D, Cannon M, et al. Physiological time series: distinguishing fractal noises from motions. Pflügers Archiv - European Journal of Physiology. 2000; 439: 403-15.

36. Chen C, Jin Y, Lo IL, Zhao H, Sun B, Zhao Q, et al. Complexity Change in Cardiovascular Disease. Int J Biol Sci. 2017; 13: 1320-8.

37. Sun S, Jin Y, Chen C, Sun B, Cao Z, Lo IL, et al. Entropy Change of Biological Dynamics in Asthmatic Patients and Its Diagnostic Value in Individualized Treatment: A Systematic Review. Entropy. 2018; 20: 402.

38. Jin Y, Chen C, Cao Z, Sun B, Lo IL, Liu TM, et al. Entropy change of biological dynamics in COPD. International journal of chronic obstructive pulmonary disease. 2017; 12: 2997-3005.

39. Dong J, Jing B, Ma X, Liu H, Mo X, Li H. Hurst Exponent Analysis of Resting-State fMRI Signal Complexity across the Adult Lifespan. Frontiers in Neuroscience. 2018; 12.34

40. Xiong W, Faes L, Ivanov PC. Entropy measures, entropy estimators, and their performance in quantifying complex dynamics: Effects of artifacts, nonstationarity, and long-range correlations. Physical Review E. 2017; 95: 062114 . 